\title{
Recomendações sobre a Utilização de Fármacos \\ Psicotrópicos durante a Pandemia COVID-19
}

\author{
Recommendations about the Use of Psychotropic \\ Medications during the COVID-19 Pandemic
}

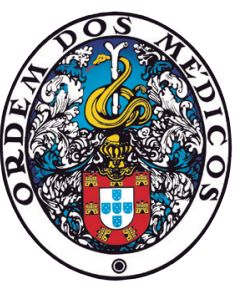

\author{
Gabriela ANDRADE $\triangle^{1,2}$, Frederico SIMÕES DO COUTO ${ }^{1,3}$, Luis CÂMARA PESTANA ${ }^{1,2}$ \\ Acta Med Port 2020 Oct;33(10):693-702 - https://doi.org/10.20344/amp.13976
}

\section{RESUMO}

Introdução: A pandemia de COVID-19 constitui uma ameaça particularmente relevante para os portadores de doença mental e um novo desafio para os profissionais que os acompanham. Até à data, tanto quanto sabemos, não existe qualquer revisão abrangente publicada relativamente à utilização de fármacos psicotrópicos durante a pandemia COVID-19.

Material e Métodos: Revisão não sistemática da literatura. A pesquisa na PubMed foi realizada com os termos 'psychotropic drugs', 'COVID-19', 'psychiatry' e 'pandemic'. Foram incluídos os consensos e as normas publicadas pelas sociedades científicas, entidades governamentais e agências regulamentares de medicamentos.

Resultados e Discussão: Apresentam-se recomendações relativamente à utilização de psicofármacos durante a pandemia COVID-19, em contexto de ambulatório e de internamento. O tratamento da perturbação afetiva bipolar e da esquizofrenia tem agora dificuldades acrescidas. Alguns psicofármacos interferem com os mecanismos fisiopatológicos envolvidos na infeção pelo novo coronavírus e têm interações com os fármacos utilizados no tratamento da COVID-19. Em doentes com COVID-19 e com delirium, a utilização de psicofármacos poderá ser necessária. A cessação tabágica altera os níveis séricos de alguns psicofármacos e pode condicionar a sua utilização.

Conclusão: A pandemia de COVID-19 coloca novos desafios na prática clínica. Os doentes psiquiátricos constituem uma população vulnerável, sendo frequentemente necessária uma avaliação clínica, laboratorial e eletrocardiográfica cuidadosa, naqueles com o diagnóstico de COVID-19. Os doentes mentais com COVID-19 apresentam uma complexidade acrescida na gestão da sua terapêutica habitual.

Palavras-chave: COVID-19; Fármacos Psicotrópicos; Pandemia; Psiquiatria

\section{ABSTRACT}

Introduction: The COVID-19 pandemic is a particularly relevant threat to mentally ill patients, and it constitutes a new challenge for health care providers. To the best of our knowledge, there is not any embracing published review about the use of psychotropic drugs during the COVID-19 pandemic.

Material and Methods: Non-systematic literature review. A search in the PubMed database was performed, with the terms 'psychotropic drugs', 'COVID-19', 'psychiatry' and 'pandemic'. Consensus and clinical guidelines about psychotropic drugs and COVID-19 approach, published by scientific societies, governmental entities and drug regulatory agencies were included.

Results and Discussion: We present the recommendations about the use of psychotropic drugs during the COVID-19 pandemic, in the outpatient and inpatient settings. The treatment of affective bipolar disorder and schizophrenia have now added increased difficulties. Some psychotropic drugs interfere with the pathophysiology of the novel coronavirus infection and they could interact with the drugs used in the treatment of COVID-19. Some patients will need pharmacological interventions due to the presence of delirium. Smoking cessation changes the serum levels of some psychotropic drugs and may influence their use.

Conclusion: The COVID-19 pandemic has created new challenges in clinical practice. Psychiatric patients are a vulnerable population and often a careful clinical, laboratorial and electrocardiographic evaluation may be needed, particularly in those diagnosed with COVID-19. The regular treatment of mentally ill patients with COVID-19 presents increased complexity.

Keywords: COVID-19; Pandemics; Psychiatry; Psychotropic Drugs

\section{INTRODUÇÃO}

A pandemia de COVID-19 representa uma ameaça particularmente relevante para os doentes mentais e um novo desafio para os profissionais que os acompanham. É admissível que estes doentes tenham uma maior vulnerabilidade para a infeção COVID-19, atendendo às características biológicas (elevado número de fumadores com compromisso respiratório e ao risco de desenvolvimento de complicações associadas às síndromas metabólicas), às psicológicas (má adesão ao confinamento, incumprimento de regras) e sociais (compromisso social, pobreza, limitação do acesso precoce a cuidados de saúde).
Aproximadamente $14 \%$ dos doentes com COVID-19 desenvolvem doença grave com necessidade de hospitalização ${ }^{1}$ e $5 \%$ podem apresentar critérios de admissão em unidades de cuidados intensivos (UCl)..$^{1,2}$ É de antecipar que possam vir a existir doentes mentais infetados que necessitem quer de internamento em unidades de Medicina/ $\mathrm{UCl}$, atendendo à gravidade da doença, quer de internamento prioritariamente psiquiátrico em área dedicada ao COVID-19 (ADC) hospitalar.

Até à data não se encontrou nenhuma revisão abrangente relativa à utilização de psicofármacos durante a

1. Serviço de Psiquiatria e Saúde Mental, Centro Hospitalar Universitário Lisboa Norte. Lisboa. Portugal.

2. Clínica Universitária de Psiquiatria e Psicologia Médica. Faculdade de Medicina. Universidade de Lisboa. Lisboa. Portugal.

3. Instituto de Farmacologia e Neurociências. Faculdade de Medicina. Universidade de Lisboa. Lisboa. Portugal.

$\triangle$ Autor correspondente: Gabriela Andrade. gabriela.andrade@chln.min-saude.pt

Recebido: 23 de abril de 2020 - Aceite: 16 de junho de 2020 | Copyright $\odot$ Ordem dos Médicos 2020 
pandemia COVID-19. Pretende-se rever as recomendações relativas à abordagem psicofarmacológica, durante a pandemia, dos doentes mentais, independentemente de estarem infetados ou não.

\section{MATERIAL E MÉTODOS}

Foi efetuada uma revisão não sistemática da literatura com o objetivo de pesquisar artigos científicos sobre o uso de fármacos psicotrópicos durante a pandemia COVID-19. Os autores identificaram resumos e textos completos da base de dados PubMed até 19 de abril de 2020. Os termos MeSH usados na pesquisa foram: 'psychotropic drugs', 'COVID-19', 'psychiatry' e 'pandemic'. Adicionalmente, foram revistas as referências dos estudos encontrados para identificar eventuais publicações complementares.

Foram, também, incluídos consensos e normas de orientação clínica sobre fármacos psicotrópicos e a abordagem da infeção COVID-19. Estes documentos foram publicados por sociedades científicas (Royal College of Psychiatrists e American Psychiatric Association), entidades governamentais [Direção-Geral da Saúde (DGS) e National Health Service] e agências regulamentares de medicamentos de Portugal, do Reino Unido e dos Estados Unidos da América. A extração de dados foi efetuada independentemente por dois dos investigadores.

\section{RESULTADOS E DISCUSSÃO}

\section{Abordagem dos portadores de doença mental com CoVID-19}

A norma da DGS n. ${ }^{\circ}$ 011/2020 introduziu o modelo de intervenção em serviços de Psiquiatria e Saúde Mental, nas várias vertentes assistenciais. ${ }^{3} \mathrm{~A}$ referida norma advoga que doentes mentais com COVID-19 deverão ser transferidos para uma enfermaria COVID-19 (com apoio da Psiquiatria de Ligação), pelo risco potencial de contágio a outros doentes e a profissionais e risco de descompensação não psiquiátrica da doença. ${ }^{3}$ Contudo, a decisão sobre o serviço ou unidade em que o doente deverá ser internado e também o nível de cuidado compete ao responsável pela ADC hospitalar. ${ }^{3}$

Nas situações em que o internamento de um doente mental com COVID-19 observado em ADC não seja possível, poderá ser necessária a prestação de cuidados em enfermaria de Psiquiatria na ausência de critérios de gravidade associados à infeção (Fig.1).

As orientações resultantes da nossa pesquisa destinam-se ao tratamento psicofarmacológico de doentes internados e em ambulatório durante a pandemia COVID-19, sendo que o tratamento da perturbação afetiva bipolar (PAB) (Fig. 2) e da esquizofrenia (Fig. 3) poderá revelar dificuldades acrescidas.

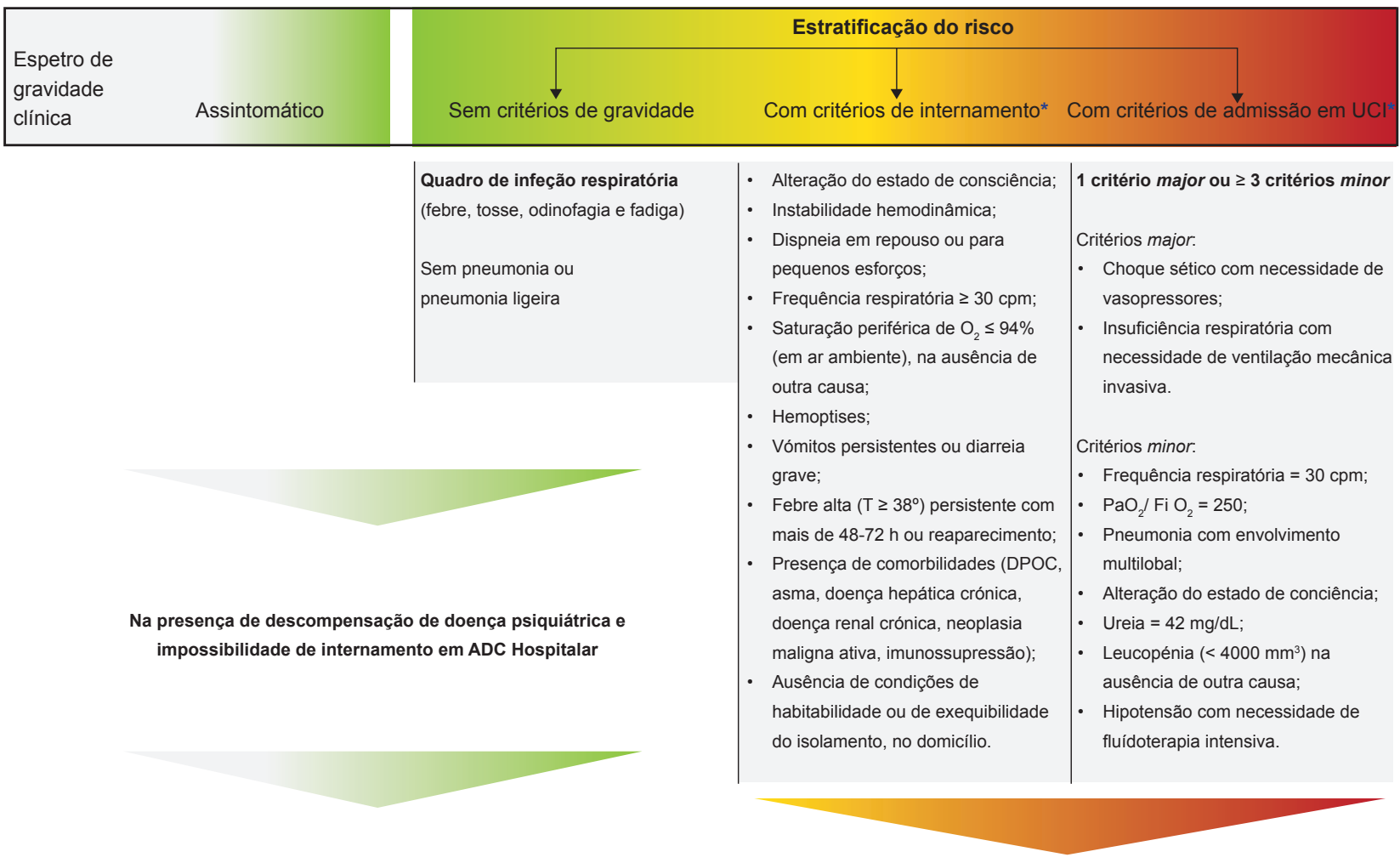

Figura 1 - Abordagem do doente COVID-19 com patologia psiquiátrica, de acordo com a gravidade clínica *: adaptado de 2,3

ADC: áreas dedicadas COVID-19; DPOC: doença pulmonar obstrutiva crónica; $\mathrm{FiO}_{2}$ : fração inspirada de oxigénio; $\mathrm{PaO}_{2}$ : pressão parcial de oxigénio no sangue arterial; UCI: unidade de cuidados intensivos 


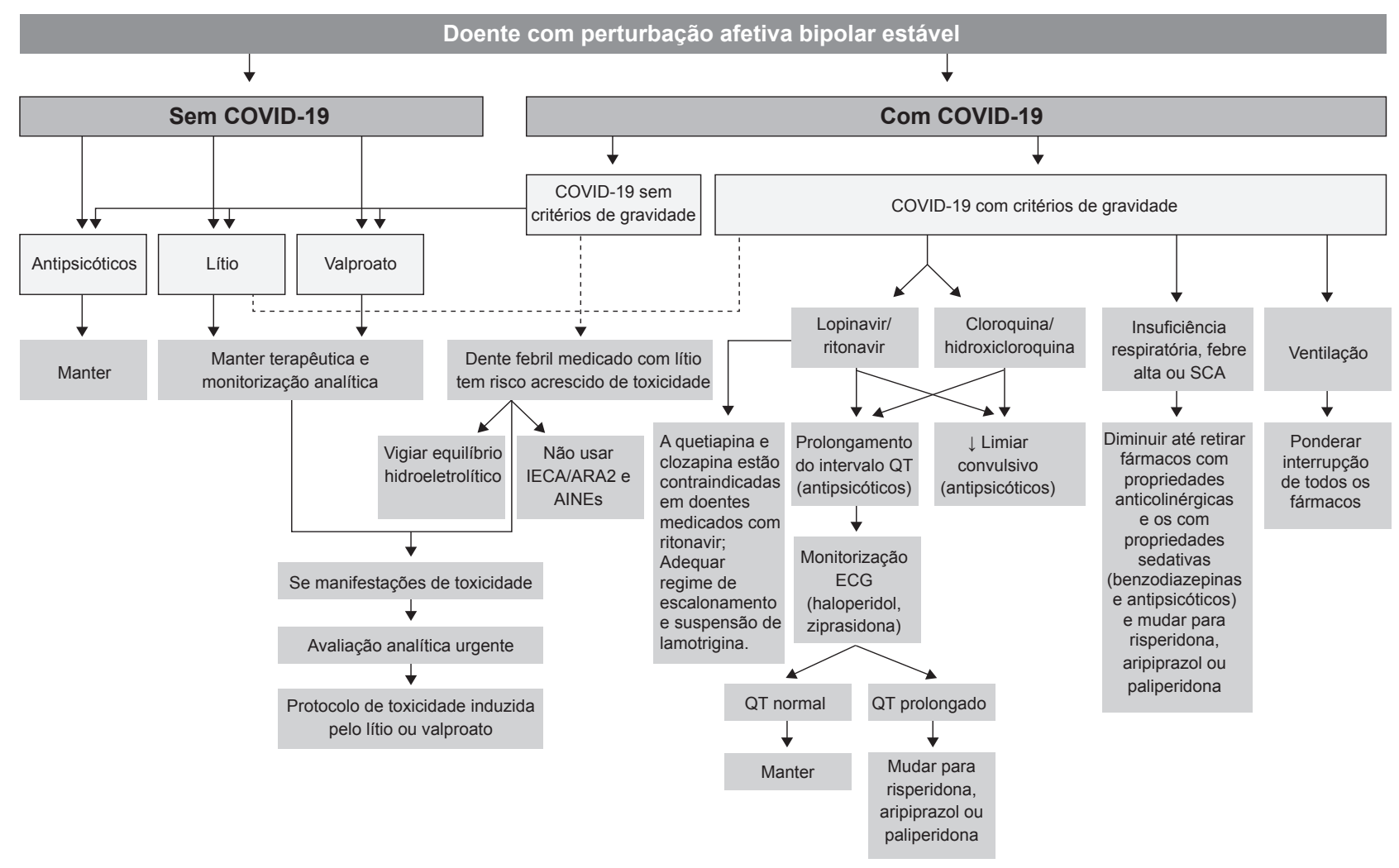

Figura 2 - Abordagem do doente com PAB durante a pandemia COVID-19

AINEs: anti-inflamatórios não esteroides; ARA2: antagonistas dos recetores da angiotensina II; SCA: síndrome confusional aguda

\section{Recomendações quanto ao uso de psicofármacos (em doentes infetados e não infetados) \\ Lítio}

A principal indicação do lítio é no tratamento da PAB. ${ }^{4}$ Nos doentes com episódios maníacos decorrentes de PAB é recomendável o início do tratamento em monoterapia ou a utilização alternativa de antipsicóticos de segunda geração. Nos doentes com episódio maníaco que responderam ao tratamento com antipsicóticos, a sua utilização estará indicada para a prevenção das recorrências.

O lítio apresenta uma janela terapêutica estreita, pelo que a sua monitorização está recomendada para excluir a existência de níveis plasmáticos superiores a $1,5 \mathrm{mmoL} / \mathrm{L}$ que, geralmente, estão associados a toxicidade. ${ }^{5} \mathrm{~A}$ determinação dos seus níveis deve ser realizada, idealmente, 12 horas após a última administração. ${ }^{6}$ Existem vários fatores que conduzem a um aumento da sua concentração plasmática, nomeadamente, hiponatremia, desidratação e interação com inibidores da enzima conversora de angiotensina II (IECA), antagonistas dos recetores de angiotensina II (ARA2), diuréticos tiazídicos e anti-inflamatórios não esteroides (AINEs). ${ }^{5,6}$ Estes fármacos podem aumentar a concentração de lítio em até quatro vezes o seu valor habitual. ${ }^{5}$

O lítio está contraindicado na insuficiência renal grave e na insuficiência cardíaca. ${ }^{4}$ Níveis tóxicos de lítio podem estar associados a alterações de gravidade variável a nível gastrointestinal, cardiovascular e no sistema nervoso central (SNC), podendo, nalguns casos, conduzir à morte. ${ }^{4} \mathrm{~A}$ redução da dose diária ou a interrupção do lítio deverá ser considerada na presença de sinais de toxicidade. O tratamento de eleição na intoxicação grave por lítio é a hemodiálise ${ }^{4}$, indicado quando a litemia está acima de 2,5 a 3 $\mathrm{mmoL} / \mathrm{L}^{5,6}$ e existe deterioração clínica (alterações neurológicas graves, compromisso cardiovascular e insuficiência respiratória). ${ }^{4,5}$

Os doentes com COVID-19 poderão apresentar um risco aumentado de desenvolver toxicidade induzida pelo lítio, através de diversos mecanismos. O estado febril está associado a mecanismos periféricos em resposta ao aumento de temperatura desencadeado pelo centro termorregulador, nomeadamente, vasodilatação, sudorese e consequente desidratação e hipovolémia. ${ }^{78}$ Adicionalmente, os doentes podem estar medicados com AINEs ou com outros fármacos com potencial de interação (havendo alguma evidência de que a furosemida possa ser mais segura do que a hidroclorotiazida) ${ }^{4,9}$

Todavia, se a necessidade da administração de fármacos com potencial de interação for imperiosa, recomenda-se essa administração na menor dose possível, bem como a realização do doseamento do lítio antes e de forma regular durante esse período. Deve ser evitada a prescrição destes fármacos de forma intermitente, pois as oscilações das concentrações são maiores e imprevisíveis.

A avaliação do risco-benefício relativamente à instituição e manutenção da terapêutica com lítio é efetuada em função de cada doente, tendo presente que a sua interrupção súbita está associada a aumento do risco de recaída e que a sua eficácia pode ficar comprometida (Fig. 2). ${ }^{6}$ 


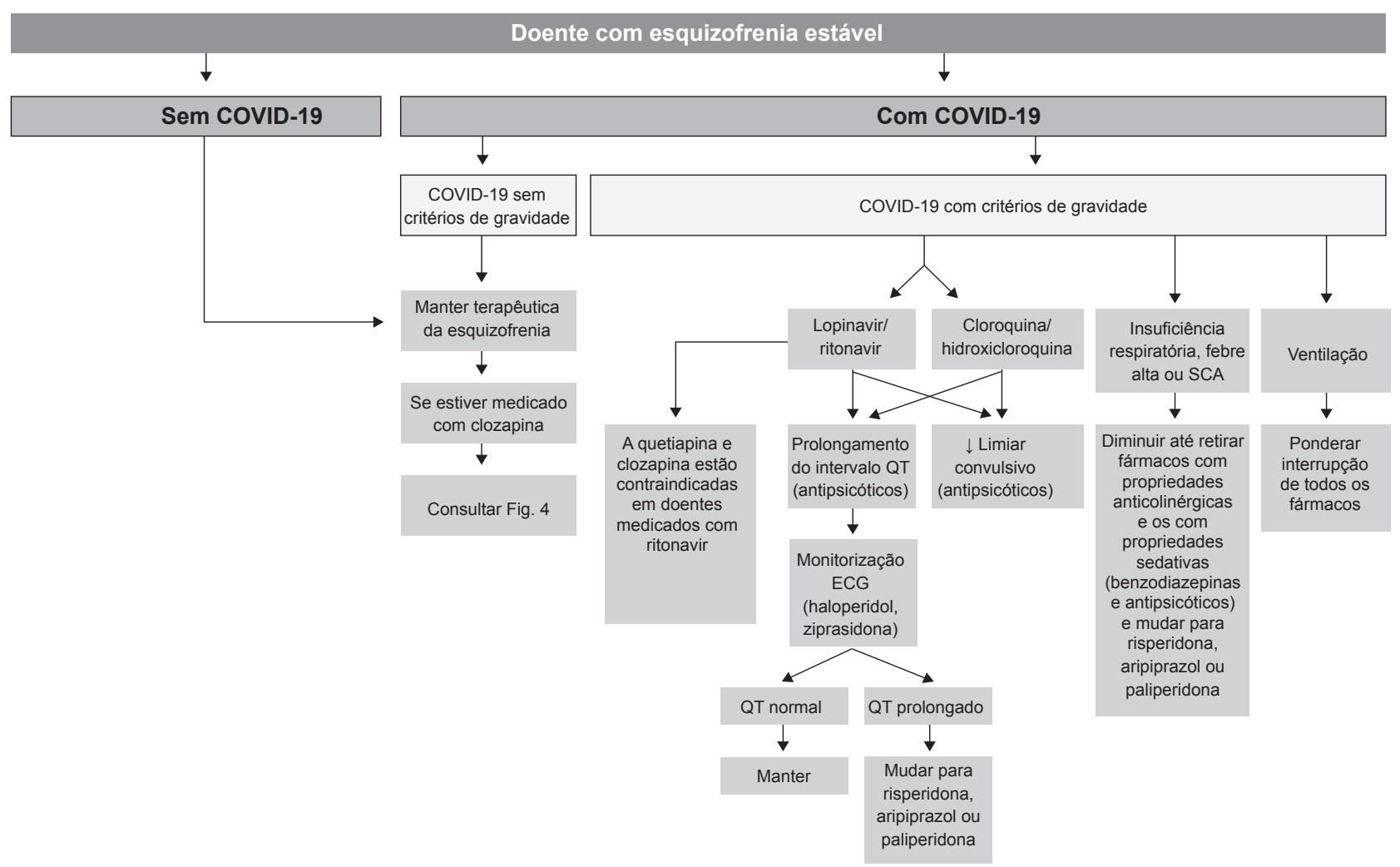

Figura 3 - Abordagem do doente com esquizofrenia durante a pandemia COVID-19

\section{Valproato de sódio}

É reconhecida a indicação do valproato de sódio (antiepilético e anticonvulsivante) no tratamento do episódio maníaco associado à $\mathrm{PAB}$, quando o lítio está contraindicado ou não é tolerado. ${ }^{10}$ Está também indicado na terapêutica de manutenção em doentes com episódio de mania aguda anterior que responderam ao tratamento com valproato. ${ }^{10}$

O valproato de sódio apresenta uma janela terapêutica mais ampla comparativamente ao lítio. ${ }^{5}$ No contexto da pandemia, os efeitos adversos que se consideram mais relevantes são a existência de alterações do perfil hepático (frequentemente, transitórias e, raramente, associadas a hepatite fulminante), leucopenia e trombocitopénia. ${ }^{6}$ Muitos dos efeitos adversos relacionam-se com a sua concentração plasmática elevada. A frequência e gravidade das manifestações está associada a níveis séricos superiores a $100 \mathrm{mg} / \mathrm{dL} .{ }^{6}$

A disfunção hepática significativa constitui uma contraindicação do valproato de sódio. ${ }^{10}$ Este possui uma forte ligação plasmática a proteínas. Em UCl, a hipoproteinemia é comum, a ligação do valproato de sódio às proteínas plasmáticas é imprevisível e a sua concentração total não é preditora da sua fração livre (mesmo corrigida para a albumina), ${ }^{11}$ pelo que se sugere uma monitorização da fração livre mais regular nestes doentes.

A inibição do metabolismo do valproato de sódio pelo ácido acetilsalicílico (em doses superiores a $300 \mathrm{mg} / \mathrm{dia}$ ) está descrita, bem como o aumento da concentração plasmática de varfarina quando coadministrada com este estabilizador de humor. ${ }^{6,10}$ A utilização concomitante destes fármacos deve ser feita com precaução, recomendando-se a monitorização da valproatémia.

\section{Clozapina}

O tratamento da esquizofrenia durante a pandemia COVID-19 poderá revelar-se mais complexo (Fig. 3), particularmente nos doentes medicados com clozapina.

A clozapina está indicada no tratamento da esquizofrenia resistente ao tratamento ${ }^{6,12,13}$ e em perturbações psicóticas que ocorrem durante o curso da doença de Parkinson, nos casos em que o tratamento convencional não mostrou eficácia. ${ }^{13}$

Uma das complicações mais graves associadas à clozapina é a agranulocitose, a qual pode conduzir a sépsis e ser fatal. ${ }^{7,15,17}$ Por isso, a sua utilização deverá ser limitada a doentes que apresentem inicialmente níveis leucocitários normais [contagem leucocitária superior a $3500 / \mathrm{mm}^{3}(3,5 \mathrm{x}$ $10^{9} / \mathrm{L}$ ) e contagens absolutas de neutrófilos (CAN) superiores a $\left.2000 / \mathrm{mm}^{3}\left(2,0 \times 10^{9} / \mathrm{L}\right)\right]$, e nos quais possam ser efetuadas contagens leucocitárias e CAN semanalmente nas primeiras 18 semanas de tratamento e, depois, em intervalos de, pelo menos, quatro semanas. ${ }^{13,15}$ A monitorização tem de continuar durante o tratamento e durante quatro semanas após a interrupção completa da clozapina. ${ }^{13,15} \mathrm{Em}$ bora sendo necessária a determinação dos níveis séricos de clozapina, nem todos os laboratórios têm capacidade para a realizar.

O desenvolvimento de neutropenia é um indicador de risco aumentado de agranulocitose (CAN inferior a 0,5 $\mathrm{x}$ $\left.10^{9} / \mathrm{L}\right),{ }^{14}$ não é dose-dependente e, geralmente, ocorre nas 
primeiras 18 semanas de tratamento. ${ }^{13}$ As incidências de neutropenia e de agranulocitose são de, respetivamente, $0,8 \%$ e $3 \% .^{14}$

Pelo risco de exposição à COVID-19, a realização do controlo hematológico regular pode implicar riscos acrescidos. Por outro lado, doentes em quarentena têm limitações na acessibilidade à realização de exames laboratoriais. ${ }^{16}$ A omissão transitória do controlo analítico pode ser considerada uma estratégia razoável ${ }^{12}$ em doentes medicados com clozapina há mais de um ano, sem alterações prévias no hemograma, ${ }^{17}$ e que integrem o grupo de risco associado à doença COVID-19.

A realização de uma avaliação médica urgente, incluindo a realização de hemograma, está indicada nos doentes que apresentem sintomas sugestivos de infeção respiratória (tosse, febre, odinofagia ou outros sintomas do tipo gripal). ${ }^{17-19}$

Em caso de COVID-19, sugere-se uma avaliação clínica com pesquisa de sinais de toxicidade induzida pela clozapina, a realização do hemograma completo e a determinação dos níveis séricos de clozapina. ${ }^{18}$

A redução da dose em doentes infetados sem critérios de gravidade, não fumadores, não tem evidência robusta. Alguns autores referem que a clozapina está associada a um aumento de risco de pneumonia devido à sialorreia e consequente aspiração ${ }^{17}$ e através de mecanismos imuno-mediados. ${ }^{17,20}$ Todavia, o risco de recaída da psicose é elevado após redução ou suspensão da dose, particularmente, se houver uma descontinuação súbita. ${ }^{17}$

Em doentes febris, a redução da dose para metade está indicada na presença de manifestações de toxicidade induzida pela clozapina (sedação excessiva, mioclonias e convulsões) e esta dose deverá ser mantida até ao final do terceiro dia de apirexia. ${ }^{17}$

As alterações na fórmula leucocitária dos doentes com COVID-19 são variáveis, sendo a linfopenia o achado mais comum. ${ }^{21}$ A continuação do tratamento com clozapina é segura em doentes que apresentem leucopenia associada a uma CAN normal ou ligeiramente reduzida, ${ }^{14}$ na ausência de contraindicações. A abordagem dos doentes com neutropenia está descrita na Fig. 4.

O nível sérico de clozapina pode estar aumentado devido a alterações do seu metabolismo e/ou à cessação tabágica, ${ }^{14,17,18}$ devendo-se ponderar um ajuste de dose nestes doentes.

Em doentes com depressão do SNC, compromisso da função renal ou cardíaca grave e/ou doença hepática, ${ }^{13}$ a clozapina está contraindicada. Se tiver havido descontinuação da clozapina durante um período superior a 48 horas, é

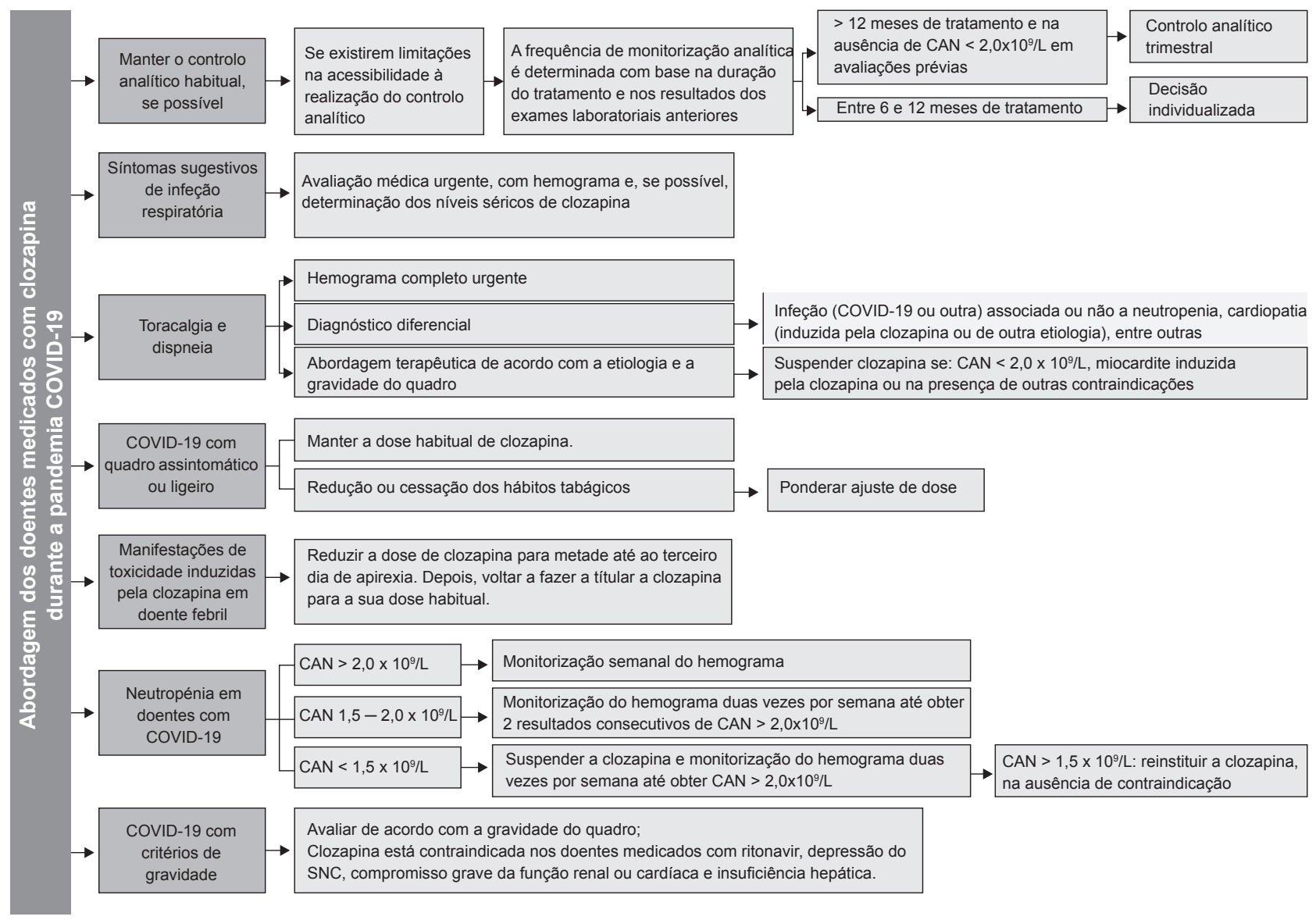

Figura 4 - Abordagem do doente medicado com clozapina durante a pandemia COVID-19§ CAN: contagem absoluta de neutrófilos; SNC: sistema nervoso central

§: adaptado $6,7,12,13,17-19,35,40$ 
necessário fazer de novo a titulação deste fármaco. ${ }^{18}$

Em doentes com toracalgia e dispneia, o diagnóstico diferencial deve incluir, para além da suspeita de infeção COVID-19, infeções não-COVID ${ }^{12,14}$ associadas a neutropenia e outras doenças inflamatórias não infeciosas, como a miocardite (em que a interrupção de clozapina está indicada). ${ }^{13}$ Contudo, sabe-se que doentes com COVID-19 podem apresentar cardiomiopatia. ${ }^{21}$

\section{Antipsicóticos injetáveis de libertação prolongada (APILP)}

A periodicidade de administração de APILP depende da substância ativa. Nas situações em que não existe possibilidade de administrar o APILP da forma e na frequência habituais, podem ser consideradas várias estratégias. ${ }^{18,22}$ Sempre que possível, sugere-se a escolha de um APILP com um maior intervalo de administração.

Em doentes estáveis medicados quinzenalmente com solução injetável de risperidona de libertação prolongada, sugere-se a mudança para palmitato de paliperidona na dose equivalente, atendendo à sua maior semi-vida. ${ }^{22}$ Nos doentes medicados com injetável mensal de palmitato de paliperidona, com estabilidade clínica há, pelo menos, seis meses, sugere-se a formulação de administração trimestral, na dose equivalente. ${ }^{22}$

Após alteração do esquema terapêutico, o doente deverá ser reavaliado uma semana depois. ${ }^{22}$

Em doentes com suspeita ou diagnóstico de infeção por COVID-19, a administração deverá ser feita, preferencialmente, no domicílio, pelo profissional de saúde, com equipamento de proteção individual (EPI) adequado.

Em situações particulares, pode ser diferida a administração do APILP18 e optar-se pela formulação oral (por exemplo, num doente com critérios de gravidade).

Os resumos das caraterísticas de cada um destes medicamentos (RCM) contêm orientações relativas a situações em que existe a omissão da administração prevista do APILP.

\section{Benzodiazepinas}

As benzodiazepinas estão aprovadas no tratamento sintomático da ansiedade e da insónia. ${ }^{23}$ Também podem ser utilizadas no tratamento da abstinência alcoólica ou como coadjuvantes no tratamento de outras perturbações psiquiátricas, particularmente, em quadros de descompensação que motivam internamento. Apresentam um elevado índice terapêutico, sendo que doses terapêuticas podem produzir sedação ligeira, geralmente, sem efeito depressor do centro respiratório e sem compromisso cardiovascular. ${ }^{24}$

As benzodazepinas diferem entre si pelas suas propriedades farmacocinéticas. Estão contraindicadas na presença de insuficiência respiratória grave. ${ }^{23}$ Situações de depressão respiratória ocorrem quando se verifica a combinação das benzodiazepinas com outras substâncias depressoras do SNC (nomeadamente, antidepressivos tricíclicos, opióides ou álcool).

Em doentes com alterações hepáticas, genericamente, as benzodiazepinas devem ser utilizadas com precaução e está recomendada a prescrição de oxazepam, lorazepam ou temazepam. Estes fármacos são metabolizados apenas por conjugação (reações de fase II), não sofrendo processo de metabolismo hepático de fase I (oxidação, hidroxilação ou outras). ${ }^{25}$ Para doentes com depuração renal inferior a $10 \mathrm{~mL} / \mathrm{min}$, sugere-se a redução da dose em $25 \%$ a $50 \%{ }^{25}$ Doentes idosos, com doença neurológica ou perturbação da personalidade têm risco acrescido de apresentarem reações paradoxais.

$\mathrm{Na}$ presença de repercussões sistémicas associadas a COVID-19 em doentes sob terapêutica benzodiazepínica, podem colocar-se problemas relacionados com o surgimento de um quadro de privação ou a existência de insuficiência respiratória, com eventual necessidade de admissão em UCl.

No contexto de UCl, a evidência atual recomenda a sedação com recurso a fármacos não benzodiazepínicos, em doentes com ventilação mecânica invasiva (grau de recomendação 2B). ${ }^{26} \mathrm{~A}$ utilização de benzodiazepinas em UCI está associada a piores outcomes, ${ }^{27}$ em termos de sedação excessiva, desenvolvimento de delirium, extubações mais tardias e permanência mais prolongada nestas unidades. ${ }^{28}$

\section{Opióides (buprenorfina e metadona)}

Os fármacos opióides são usados para terapia de substituição, em manutenção, dos doentes adultos com perturbação da dependência de opióides. A depressão respiratória surge essencialmente por diminuição da sensibilidade do centro respiratório ao $\mathrm{CO}_{2}$. Provavelmente os pacientes que sofrem de dependência a opióides estão em risco aumentado de ser infetados. ${ }^{29}$

Não se encontraram referências específicas para os riscos de insuficiência respiratória em doentes COVID-19 medicados com opióides. Assim, deverá prevalecer o sentido clínico individual. Os opióides devem ser usados com precaução em doentes com reserva respiratória limitada (asma, doença pulmonar obstrutiva crónica ou outras). Devem-se evitar aumentos da dose e vigiar cuidadosamente a saturação de oxigénio e os sinais de dificuldade respiratória. A dose deve ser reduzida, tanto quanto possível lentamente. Em emergência dever-se-ão usar os antagonistas dos recetores dos opióides, particularmente, a naloxona.

É de realçar que a abstinência a opióides, isoladamente, não conduz à morte, contrariamente à privação alcoólica. ${ }^{6}$

\section{Outros fármacos}

Para além dos fármacos referidos, alguns antidepressivos (antidepressivos tricíclicos, mirtazapina e trazodona) e alguns antipsicóticos (clorpromazina, levomepromazina, ciamemazina, clozapina, quetiapina e olanzapina) apresentam propriedades sedativas ${ }^{6}$, pelo que a sua prescrição deverá ser criteriosa.

\section{COVID-19 e manifestações neuropsiquiátricas}

As manifestações psiquiátricas num doente com uma 
determinada infeção viral podem decorrer do envolvimento primário do SNC, dos efeitos secundários produzidos pela resposta imunomediada ou, indiretamente, através de efeitos sistémicos. ${ }^{30}$ Dados recentemente publicados sugerem que o novo coronavírus tem propriedades neurotrópicas, ${ }^{31}$ podendo conduzir ao aparecimento de sintomas neuropsiquiátricos.

\section{Síndrome confusional aguda}

A síndrome confusional aguda (SCA) é uma das causas mais frequentes de manifestações psicopatológicas em doentes internados noutras especialidades, sendo mais comum nas pessoas de idade mais avançada, ${ }^{32-34}$ nos quadros de maior gravidade e na presença de défices sensoriais e demência. ${ }^{33,34} \mathrm{~A}$ desorientação, flutuação do estado de consciência e diminuição da atenção podem constituir a apresentação clínica da infeção COVID-19 em idosos, dado que que a febre é menos comum nesta população e nem todos os doentes infetados apresentam tosse ou dispneia.
A infeção, fármacos sedativos e/ou anticolinérgicos e exacerbação de doença crónica são alguns fatores que podem estar presentes num doente com COVID-19 e que são precipitantes da SCA. ${ }^{34}$ Este quadro pode ocorrer sob a forma hiperativa, hipoativa (mais comum e associada a pior prognóstico) ou mista. ${ }^{34}$

Nos doentes com SCA importa pesquisar a existência de etiologias metabólicas, tóxicas e farmacológicas, entre outras.

As intervenções de primeira linha são dirigidas à causa da $\mathrm{SCA}^{34}$ e utilizam-se, preferencialmente, estratégias não farmacológicas na gestão das alterações do comportamento. ${ }^{34}$ Contudo, a sua implementação na população de doentes COVID-19 é limitada. A utilização de EPI e o isolamento podem conduzir ao agravamento do quadro. ${ }^{33}$

No tratamento da SCA em doentes COVID-19 sugere-se a utilização de haloperidol (0,5 - 2 mg; máximo: 3 $5 \mathrm{mg} / \mathrm{dia})$, risperidona $(0,25$ - 0,5 mg; máximo $3 \mathrm{mg} / \mathrm{dia})$, quetiapina (12,5 - 50 mg; máximo: 50 - $100 \mathrm{mg} / \mathrm{dia})$, olanzapina (2,5 - $5 \mathrm{mg}$; máximo: 10 - $20 \mathrm{mg} / \mathrm{dia})$ e lorazepam

Tabela 1 - Interações entre os fármacos utilizados no tratamento do COVID-19 e alguns psicofármacos ${ }^{\S}$

\begin{tabular}{|c|c|c|}
\hline & Lopinavir/ ritonavir & Cloroquina/ hidroxicloroquina \\
\hline Haloperidol & $\begin{array}{l}\text { Aumenta os níveis séricos do antipsicótico por } \\
\text { inibição do CYP2D6 } \\
\text { Risco de prolongamento do intervalo QT }\end{array}$ & $\begin{array}{l}\text { Aumenta os níveis séricos ou o efeito do antipsicótico } \\
\text { Risco moderado de prolongamento do intervalo QT } \\
\text { Diminuição do limiar convulsivo. }\end{array}$ \\
\hline Clorpromazina & \multicolumn{2}{|c|}{$\begin{array}{l}\text { Aumenta os níveis séricos do antipsicótico por inibição do CYP2D6 } \\
\text { Risco de prolongamento do intervalo QT } \\
\text { Diminuição do limiar convulsivo. }\end{array}$} \\
\hline Quetiapina & $\begin{array}{l}\text { Aumenta os níveis séricos do antipsicótico por } \\
\text { inibição do CYP3A4. } \\
\text { Risco de prolongamento do intervalo QT. } \\
\text { A utilização de quetiapina está contraindicada. }\end{array}$ & $\begin{array}{l}\text { Risco moderado de prolongamento do intervalo QT } \\
\text { Diminuição do limiar convulsivo. }\end{array}$ \\
\hline Risperidona & $\begin{array}{l}\text { Aumenta os níveis séricos do antipsicótico por } \\
\text { inibição do CYP2D6 } \\
\text { Risco de prolongamento do intervalo QT }\end{array}$ & $\begin{array}{l}\text { Risco moderado de prolongamento do intervalo QT } \\
\text { Diminuição do limiar convulsivo. }\end{array}$ \\
\hline Olanzapina & Diminuição dos níveis de olanzapina. & $\begin{array}{l}\text { Risco moderado de prolongamento do intervalo QT } \\
\text { Diminuição do limiar convulsivo. }\end{array}$ \\
\hline Clozapina & $\begin{array}{l}\text { Aumenta os níveis séricos de antipsicótico por } \\
\text { inibição do CYP3A4. } \\
\text { A utilização de clozapina está contraindicada. }\end{array}$ & Diminuição do limiar convulsivo. \\
\hline Lítio & Desconhecidas & Risco moderado de prolongamento do intervalo QT \\
\hline Valproato de sódio & $\begin{array}{l}\text { Redução dos níveis séricos de valproato de } \\
\text { sódio. }\end{array}$ & $\begin{array}{l}\text { Redução da eficácia do valproato de sódio em doentes } \\
\text { com epilepsia. }\end{array}$ \\
\hline Lamotrigina & $\begin{array}{l}\text { Inibição da glucuronidação da lamotrigina. Utilizar } \\
\text { o regime de escalonamento e suspensão da } \\
\text { lamotrigina de acordo com o seu RCM. }\end{array}$ & $\begin{array}{l}\text { Redução da eficácia da lamotrigina em doentes com } \\
\text { epilepsia. }\end{array}$ \\
\hline Benzodiazepinas & \multicolumn{2}{|c|}{ Oxazepam, lorazepam e temazepam sem interações conhecidas. } \\
\hline Buprenorfina & $\begin{array}{l}\text { Aumento dos níveis de buprenorfina por inibição } \\
\text { do CYP3A4. }\end{array}$ & Desconhecidas \\
\hline Metadona & $\begin{array}{l}\text { Diminuem o efeito da metadona por indução } \\
\text { do seu metabolismo e da bomba glicoproteíca } \\
\text { de remoção de metadona do SNC. Risco de } \\
\text { precipitação de síndrome de abstinência. }\end{array}$ & Risco de prolongamento do intervalo QT \\
\hline
\end{tabular}

\$: adaptado $4,10,13,36-48$ 
(0,25 - $1 \mathrm{mg}$; máximo: $2 \mathrm{mg} / \mathrm{dia}) .^{26,32-34}$ No entanto, nem todos os doentes poderão responder a estas estratégias, pelo que se torna necessária a articulação com a Psiquiatria de Ligação.

Está recomendada a utilização de um antipsicótico na menor dose possível. O haloperidol é, geralmente, um dos fármacos de primeira linha, devendo ser utilizado com precaução pelo risco de desenvolvimento de sintomas extrapiramidais (SEP) e pelo seu potencial arritmogénico. Em pacientes com doenças neurológicas com sintomas motores, sugere-se a escolha da quetiapina, a qual apresenta um perfil de menor risco de desenvolvimento de SEP. $\mathrm{Na}$ doença de Corpos de Lewy pode haver um aumento da sensibilidade aos antipsicóticos, com consequente necessidade de escolha adequada do antipsicótico e de ajuste da sua dose.

\section{Psicofármacos e medicamentos usados no trata- mento da COVID-19}

Atualmente, em Portugal, não existem fármacos com indicação regulamentar no tratamento da doença provocada pelo novo coronavírus. Recentemente, o Infarmed recomendou a suspensão da utilização de hidroxicloroquina, ${ }^{35}$ pelo risco de desenvolvimento de efeitos adversos potencialmente graves. De qualquer forma, estão descritas interações entre algumas das potenciais intervenções medicamentosas no tratamento da doença COVID-19 e vários psicofármacos (Tabela 1), ${ }^{36-39}$ nomeadamente, no que respeita ao prolongamento do intervalo QT e à redução do limiar convulsivo.

O risco de prolongamento do intervalo QT e de torsades de pointes está aumentado se duração do intervalo QT corrigido for superior a $450 \mathrm{~ms}$ nos homens e a $470 \mathrm{~ms}$ nas mulheres. ${ }^{6}$ Nos doentes COVID-19 medicados com fármacos associados ao prolongamento do intervalo QT e com outros fatores de risco (doença cardíaca e distúrbios hidroeletrolíticos), recomenda-se uma monitorização eletrocardiográfica mais frequente.

A redução do limiar convulsivo está descrita quando há coadministração de lopinavir/ ritonavir e valproato (por redução dos seus níveis séricos) ${ }^{40}$ e nos doentes medicados com cloroquina ou hidroxicloroquina. ${ }^{37}$ Os antipsicóticos podem também diminuir o limiar convulsivo, pelo que a sua administração com fármacos com potencial de interação deverá ser feita com precaução.

A utilização de clozapina e quetiapina está contraindicada em doentes medicados com ritonavir, devido ao aumento das suas concentrações plasmáticas e risco de toxicidade associada.$^{36} \mathrm{O}$ lopinavir e ritonavir são indutores da glucuronidação da lamotrigina, pelo que em doentes medicados com este psicofármaco dever-se-á ter em consideração os regimes de escalonamento ou suspensão propostos no RCM. ${ }^{41}$

Apesar da administração lopinavir/ ritonavir estar associada a um aumento dos níveis séricos da buprenorfina, a relevância clínica deste aspeto tem sido questionada e vários autores referem não parecer haver necessidade de alteração da dose. ${ }^{42,43}$ Recomenda-se, assim, vigilância e prudência na associação, ponderando mudanças de dose de acordo com o eventual surgimento de efeitos tóxicos.

Os eventos adversos psiquiátricos mais importantes associados à terapêutica da infeção COVID-19, pela sua frequência ou gravidade, são: os comportamentos suicidários (raros) e sintomas psicóticos com a hidroxicloroquina ${ }^{44}$; a ansiedade com o lopinavir/ ritonavir ${ }^{45}$ e as manifestações psicóticas ou maniformes com glucocorticóides. ${ }^{5}$

\section{Outras considerações}

\section{Psicofármacos e termorregulação}

Durante um quadro infeccioso, pode ocorrer a perda de equilíbrio entre os mecanismos de termogénese e termólise (como a vasodilatação e a sudorese),${ }^{46}$ o que pode associar-se à SCA.

A transmissão colinérgica a nível do hipotálamo e a transmissão dopaminérgica na área pré-ótica e no hipotálamo anterior têm um papel no centro termorregulador. 47,48 Alterações na transmissão colinérgica a nível central, mas também a nível dos recetores muscarínicos M2 das glândulas sudoríparas, interferem na termorregulação. ${ }^{48}$ Alguns fármacos podem ainda causar febre através da estimulação da resposta imune ou por possuírem propriedades pirogénicas intrínsecas. ${ }^{47}$

As principais classes de psicofármacos com ações anticolinérgicas ${ }^{47,48}$ que poderão agravar estados febris são os antipsicóticos (particularmente as fenotiazinas alifáticas e piperidínicas, a clozapina, e antipsicóticos de segunda geração tais como a olanzapina e a quetiapina), alguns antidepressivos (nomeadamente os antidepressivos tricíclicos) e os fármacos utilizados no tratamento de SEP (cloridrato de biperideno e cloridrato de triexifenidilo). Sugere-se uma utilização criteriosa destes fármacos, particularmente nos doentes com critérios de gravidade.

\section{Psicofármacos e cessação tabágica}

O consumo de tabaco está relacionado com a indução de enzimas hepáticas, particularmente do CYP1A2. ${ }^{12}$ Durante a pandemia, alguns doentes poderão reduzir ou cessar os seus consumos, o que poderá levar ao aumento da concentração plasmática de alguns fármacos. Aconselha-se a avaliação clinico-laboratorial, podendo ser necessária uma redução da dose de alguns psicofármacos (nomeadamente clozapina, olanzapina e fluvoxamina). É pouco provável que a terapêutica de substituição da nicotina seja uma alternativa terapêutica, uma vez que esta não parece induzir o metabolismo hepático. ${ }^{18}$

\section{CONCLUSÃO}

A pandemia de COVID-19 coloca novos desafios na prática clínica. Os doentes psiquiátricos constituem uma população vulnerável, sendo frequentemente necessária uma avaliação clínica, laboratorial e eletrocardiográfica cuidadosa, particularmente naqueles com o diagnóstico de COVID-19. Os doentes mentais com COVID-19 apresentam uma complexidade acrescida na gestão da sua 
terapêutica habitual.

Estas recomendações pretendem ser úteis e têm como objetivo apresentar o racional clínico subjacente às estratégias psicofarmacológicas, de forma a minimizar os riscos associados.

\section{REFERÊNCIAS}

1. World Health Organization. Clinical management of severe acute respiratory infection when novel coronavirus (nCoV) infection is suspected. 2020. [consultado 2020 abr 19]. Disponível em: https:// www.who.int/publications-detail/clinical-management-of-severe-acuterespiratory-infection-when-novel-coronavirus-(ncov)-infection-issuspected.

2. Direção-Geral da Saúde. Norma n. ${ }^{\circ}$ 011/2020 - COVID-19: Fase de mitigação Saúde Mental. 2020. [consultado 2020 abr 19]. Disponível em: https://www.dgs.pt/directrizes-da-dgs/normas-e-circulares-normativas/ norma-n-0112020-de-18042020.aspx.

3. Direção-Geral da Saúde. Norma n. 004/2020 COVID-19: Fase de mitigação: Abordagem do Doente com Suspeita ou Infeção por SARSCoV-2. 2020. [consultado 2020 abr 14]. Disponível em: https://www. dgs.pt/directrizes-da-dgs/normas-e-circulares-normativas/norma-n0042020-de-23032020-pdf.aspx.

4. Infarmed. Lítio. 2020. [consultado 2020 abr 21]. Disponível em: http://app7.infarmed.pt/infomed/download_ficheiro.php?med_ id=30076\&tipo_doc=rcm.

5. Hirschfeld R, Bowden C, Gitlin M, Keck P, Suppes T, Thase M, et al. Practice guideline for the treatment of patients with bipolar disorder. 2005. [consultado 2020 abr 19]. Disponível em: https://psychiatryonline. org/pb/assets/raw/sitewide/practice_guidelines/guidelines/bipolar.pdf.

6. Taylor D; Barnes T; Young A. The Maudsley Prescribing Guidelines in Psychiatry. 13 ${ }^{\text {th }}$ ed. Hoboken: Wiley Blackwell; 2018.

7. Royal College of Psychiatrists. COVID-19. Providing medication. 2020. [consultado 2020 abr 14]. Disponível em: https://www.rcpsych.ac.uk/ about-us/responding-to-covid-19/responding-to-covid-19-guidance-forclinicians/community-and-inpatient-services/providing-medication.

8. Bouchama A, Knochel J. Heat Stroke. N Engl J Med. 2002;346:1978-88.

9. Saffer D, Coppen A. Furosemide: a safe diuretic during lithium therapy? J Affect Disord. 1983;5:289-92.

10. Infarmed. Valproato de sódio. 2020. [consultado 2020 abr 21]. Disponível em: http://app7.infarmed.pt/infomed/download_ficheiro. php?med_id=30076\&tipo_doc=rcm.

11. Riker R, Gagnon D, Hatton C, May T, Seder D, Stokem K, et al. Valproate protein binding is highly variable in ICU patients and not predicted by total serum concentrations: a case series and literature review. Pharmacotherapy. 2017;37:500-8.

12. Freudenreich $\mathrm{O}$, McEvoy J. Guidelines for prescribing clozapine in schizophrenia. UpToDate; 2020. [consultado 2020 abr 14]. Disponível em: https://www.uptodate.com/contents/guidelines-for-prescribingclozapine-in-schizophrenia.

13. Infarmed. Clozapina. 2020. [consultado 2020 abr 21]. Disponível em: http://app7.infarmed.pt/infomed/download_ficheiro.php?med_ id=4976\&tipo_doc $=$ rcm.

14. Royal College of Psychiatrists. Clozapine and blood dyscrasias in patients with coronavirus (COVID-19). 2020. [consultado 2020 abr 14]. Disponível em: https://www.rcpsych.ac.uk/docs/default-source/improving-care/ better-mh-policy/policy/clozapine-and-blood-dyscrasias-in-patientswith-coronavirus-(Covid-19).pdf?sfvrsn=1d28f4b_2.

15. Direção-Geral da Saúde. Norma n. ${ }^{\circ} 024 / 2011$ Utilização clínica de antipsicóticos. 2020. [consultado 2020 abr 14]. Disponível em: https:// www.dgs.pt/directrizes-da-dgs/normas-e-circulares-normativas/norman-0042020-de-23032020-pdf.aspx.

16. Careoregon.org. Portland: CareOregon. Clozapine and Covid-19. 2020. [consultado 2020 abr 14]. Disponível em: https://careoregon.org/docs/ default-source/covid-19/clozapine-and-covid-19.pdf.

17. Siskind D, Honer WG, Clark S, Correll CU, Hasan A, Howes O, et al. Consensus statement on the use of clozapine during the COVID-19 pandemic. J Psychiatry Neurosci. 2020;45:1-2.

18. South London and Maudsley National Health Service. COVID-19 and psychotropic medication. 2020. [consultado 2020 abr 14]. Disponível

\section{CONFLITOS DE INTERESSE}

Os autores declaram não ter conflitos de interesses relacionados com o presente trabalho.

\section{FONTES DE FINANCIAMENTO}

Este trabalho não recebeu qualquer tipo de suporte financeiro de nenhuma entidade no domínio público ou privado.

em: http://iris-initiative.org.uk/wordpress/wp-content/uploads/2020/03/ COVID-19-and-psychotropic-medication.pdf.

19. Taylor D. Clozapine emergency protocol. 2020. [consultado $2020 \mathrm{abr}$ 14]. Disponível em: https://www.rcpsych.ac.uk/docs/default-source/ improving-care/better-mh-policy/policy/clozapine---emergencyprotocol-for-patients-on-monthly-monitoring.pdf?sfvrsn=555b86d5_2.

20. Pandarakalam J. Potential risk of COVID-19 in clozapine treated patients. Re: Covid-19: outbreak could last until spring 2021 and see 7.9 million hospitalised in the UK. BMJ;2020;368:m1071.

21. Mclntosh K. Coronavirus disease 2019. UpToDate; 2020. [consultado 2020 abr 14]. Disponível em: https://www.uptodate.com/contents/ coronavirus-disease-2019-covid-19-epidemiology-virology-clinicalfeatures-diagnosis-and-prevention.

22. Berkshire West National Health Service. Guidance on managing LongActing Injectable antipsychotic Injections (LAls) during Covid-19 social isolation period. 2020. [consultado 2020 abr 14]. Disponível em: https:// www.berkshirewestccg.nhs.uk/media/3870/guidance-on-managinglong-acting-injectable-antipsychotic-injections-lais-during-Covid-19social-isolation-period.

23. Direção-Geral de Saúde. Tratamento sintomático da ansiedade e insónia com benzodiazepinas e fármacos análogos. Norma de orientação clínica n. ${ }^{\circ}$ 055/201. 2020. [consultado 2020 abr 14]. Disponível em: https://www.dgs.pt/directrizes-da-dgs/normas-e-circulares-normativas/ norma-n-0552011-de-27122011-jpg.aspx.

24. Becker DE. Pharmacodynamic considerations for moderate and deep sedation. Anesth Prog. 2012;59:28-42.

25. Procyshyn R, Butler K, Jeffries JJ. Clinical handbook of psychotropic drugs. 22 ${ }^{\text {nd }}$ ed. Boston: Hogrefe Publishing; 2017.

26. Barr J, Fraser GL, Puntillo K, Ely EW, Gélinas C, Dasta JF, et al. Clinical practice guidelines for the management of pain, agitation, and delirium in adult patients in the intensive care unit. Crit Care Med. 2013;41:263306.

27. Ely EW, Dittus RS, Girard TD, Skrobik Y. Should benzodiazepines be avoided in mechanically ventilated patients? Yes. Chest. 2012;142:2814.

28. Ferrell BA, Girard TD. Sedative choice: a critical decision. Am J Respir Crit Care Med. 2014;189:1295-7.

29. Sun Y, Bao Y, Kosten T, Strang J, Shi J, Lu L. Editorial: Challenges to opioid use disorders during COVID-19. Am J Addict. 2020:1-2.

30. Levenson J. The American Psychiatric Association Publishing Textbook of Psychosomatic Medicine and Consultation-Liaison Psychiatry. $3^{\text {rd }}$ ed. Washington: American Psychiatric Association Publishing; 2019.

31. Wu Y, Xu X, Chen Z, Duan J, Hashimoto K, Yang L, et al. Nervous system involvement after infection with COVID-19 and other coronaviruses. Brain Behav Immun Health. 2020 (in press). doi: 10.1016/j.bbi.2020.03031.

32. Francis J, Young GB. Diagnosis of delirium and confusional states. UpToDate; 2020. [consultado 2020 abr 14]. Disponível em: https://www. uptodate.com/contents/diagnosis-of-delirium-and-confusional-states.

33. Jenkinson J. Delirium management advice for patients with confirmed or suspected COVID-19 in the acute trust setting. Rcpsych; 2020. [consultado 2020 abr 14]. Disponível em: https://www.rcpsych.ac.uk/ docs/default-source/members/faculties/old-age/Covid-19-deliriummanagement-guidance.pdf?sfvrsn=2d5c6e63_2.

34. Marcantonio ER. Delirium in hospitalized older adults. N Engl J Med. 2017;377:1456-66.

35. Infarmed. Infarmed e DGS recomendam suspensão do uso de hidroxicloroquina em doentes com COVID-19. 2020. [consultado 2020 jun 23]. Disponível em: https://www.infarmed.pt/documents/15786/3584909/ Comunicado+de+Imprensa+-+Infarmed+e+DGS+recomendam+suspe $\mathrm{ns} \% \mathrm{C} 3 \% \mathrm{~A} 3 \mathrm{o}+\mathrm{do}+\mathrm{uso}+\mathrm{de}+$ hidroxicloroquina+em+doentes+com+COV ID-19/1254453b-5943-1668-12cc-828e2bee3ab1.

36. Electronic medicines compendium (emc). Ritonavir. 2020. [consultado 
2020 abr 21]. Disponível em: https://www.medicines.org.uk/emc/ product/510/smpc.

37. Electronic medicines compendium (emc). Hydroxychloroquine. 2020 [consultado 2020 abr 21]. Disponível em: https://www.medicines.org.uk/ emc/product/1764/smpc.

38. Medscape.com. Drug interaction checker. 2020. [consultado 2020 abr 14]. Disponível em: https://reference.medscape.com/druginteractionchecker.

39. Drugs.com. Drug interactions checker. 2020. [consultado 2020 abr 14]. Disponível em: https://www.drugs.com/drug_interactions.html.

40. Electronic medicines compendium (emc). Lopinavir/ ritonavir. 2020 [consultado 2020 abr 21]. Disponível em: https://www.medicines.org.uk/ emc/product/10479/smpc

41. Infarmed. Lamotrigina. 2020. [consultado 2020 abr 21]. Disponível em: http://app7.infarmed.pt/infomed/download_ficheiro.php?med_ id $=4878 \&$ tipo doc $=\mathrm{rcm}$

42. Bruce RD, Altice FL, Moody DE, Morse GD, Andrews L, Lin SN, et al. Pharmacokinetic interactions between buprenorphine/naloxone and once-daily lopinavir/ritonavir. J Acquir Immune Defic Syndr. 2010;54:511-
4.

43. McCance-Katz EF, Moody DE, Smith PF, Morse GD, Friedland G, Pade, et al. Interactions between buprenorphine and antiretrovirals. II. The protease inhibitors nelfinavir, lopinavir/ritonavir, and ritonavir. Clin Infect Dis. 2006;43:S235-46.

44. Infarmed. Hidroxicloraquina. 2020. [consultado 2020 abr 21]. Disponível em: http://app7.infarmed.pt/infomed/download_ficheiro.php?med id=6931\&tipo_doc=rcm

45. Infarmed. Lopinavir/ ritonavir. 2020. [consultado 2020 abr 21]. Disponível em: http://http://app7.infarmed.pt/infomed/download_ficheiro.php?med id=597811\&tipo_doc.

46. Silbernagl S, Lang F. Color atlas of pathophysiology. $3^{\text {rd }}$ ed. Stuttgart: Thieme; 2016.

47. Walter E, Carraretto M. Drug-induced hyperthermia in critical care. $J$ Intensive Care Soc. 2015;16:306-11.

48. Lee CP, Chen PJ, Chang CM. Heat stroke during treatment with olanzapine, trihexyphenidyl, and trazodone in a patient with schizophrenia. Acta Neuropsychiatr. 2015;27:380-5 